\title{
The distribution of $\gamma$-adrenoceptors and $\mathrm{P}_{2}$ purinoceptors in mesenteric arteries and veins of the guinea-pig
}

\author{
G.D.S. Hirst $\&^{1}$ P. Jobling \\ Department of Zoology, University of Melbourne, Parkville, Victoria, Australia, 3052
}

1 Membrane potential changes and contractions were recorded from mesenteric arteries and veins of the guinea-pig, during perivascular nerve stimulation or application of noradrenaline or adenosine triphosphate (ATP).

2 After $\alpha$-adrenoceptor blockade, noradrenaline activated low affinity adrenoceptors $(\gamma$-adrenoceptors) causing depolarization and arterial contraction only in the presence of an inhibitor of catecholamine uptake.

3 Noradrenaline did not cause depolarization or contraction of the vein after $\alpha$-adrenoceptor blockade even after catecholamine uptake was blocked.

4 Adenosine triphosphate caused depolarization and contraction of both arteries and veins. These responses were abolished by $\alpha$-, $\beta$-,methylene adenosine triphosphate (Me-ATP).

5 Me-ATP abolished rapid excitatory junction potentials (e.j.ps) caused by perivascular nerve stimulation of arteries but had no effect on arterial responses mediated by $\gamma$-adrenoceptors.

6 In veins, perivascular nerve stimulation evoked slow e.j.ps which persisted in the presence of Me-ATP but were abolished after blockade of $\alpha$-adrenoceptors.

7 The observations indicate that $P_{2}$ purinoceptors are present on both mesenteric artery and vein whilst $\gamma$-adrenoceptors are localized near the neuromuscular junction of the artery. However $\gamma$ adrenoceptors do not appear to be directly involved in the generation of arterial e.j.ps.

\section{Introduction}

In a number of arterial beds, a part of the response to sympathetic nerve stimulation persists in the presence of both $\alpha$ - and $\beta$-adrenoceptor antagonists. Examples are the neurally activated vasoconstrictions of rabbit mesenteric and saphenous arteries (Kugelgen \& Starke, 1985; Burnstock \& Warland, 1987), dog mesenteric arteries (Muramatsu, 1986) and the increase in peripheral resistance of rabbit hindlimbs (Hirst \& Lew, 1987).

Sympathetic nerve stimulation initiates a rapid excitatory junction potential (e.j.p.) in most mammalian systemic arteries (Hirst, 1977; Holman \& Surprenant, 1979; Surprenant, 1980; Kuriyama \& Suzuki, 1981; Kuriyama \& Suyama, 1983; Kreulen, 1986). If the e.j.p. is sufficiently large or if successive e.j.ps are summed together, the depolarization leads to the activation of voltage-dependent calcium channels and constriction follows (Hirst \& Edwards, 1988). In all systemic arteries studied, e.j.ps have

\footnotetext{
${ }^{1}$ Author for correspondence.
}

been found to be resistant to $\alpha$-adrenoceptor blockade but are abolished or reduced in amplitude by drugs interfering with neuronal release (Holman \& Surprenant, 1979; 1980; Hirst \& Neild, 1980; Cheung, 1982; Fujiwara et al., 1982; Hirst et al., 1982; Suzuki \& Kou, 1983).

Two hypotheses have been suggested to explain the non $\alpha$-adrenoceptor-mediated e.j.ps in systemic arteries. One suggests that released noradrenaline activates specialized junctional adrenoceptors termed $\gamma$-adrenoceptors which are located close to sympathetic nerve terminals (Hirst \& Neild, 1980; 1981; Hirst et al., 1982; Luff et al., 1987). The other suggests that adenosine triphosphate (ATP), or a closely related compound, co-released with noradrenaline from the nerve terminal acts on $P_{2}$ purinoceptors to produce the rapid e.j.p. (Sneddon \& Burnstock, 1984; Kügelgen \& Starke, 1985; Ishikawa, 1985). This hypothesis is supported by the finding that $\alpha-, \beta$-,methylene ATP (Me-ATP), a stable analogue of ATP desensitizes $P_{2}$ purinoceptor effects 
of ATP and blocks e.j.ps (Sneddon \& Burnstock 1984; Ishikawa, 1985).

In contrast to arteries, rapid e.j.ps are not initiated by sympathetic nerve stimulation in veins, perhaps with the exception of dog mesenteric and rat saphenous veins (Cheung, 1981; Suzuki, 1984). Rather, repetitive sympathetic nerve stimulation typically produces a depolarization which is slow in onset and very long lasting (Suzuki, 1981; Kreulen, 1986). These slow e.j.ps are associated with contraction. Both the slow e.j.p. and the contraction are abolished by $\alpha$-adrenoceptor antagonists (Suzuki, 1981; Kreulen, 1986).

The experiments described in this paper were designed to examine the distribution of $\gamma$ adrenoceptors and purinoceptors on mesenteric arteries and veins. The nature of the response to sympathetic nerve stimulation was also determined. It was found that, whereas $\gamma$-adrenoceptors were restricted to mesenteric arteries, $\mathbf{P}_{2}$ receptors were present on both arterial and venous tissues. $\gamma$ Adrenoceptor responses in mesenteric arteries persisted in the presence of Me-ATP; $\gamma$-adrenoceptors are therefore unlikely to be involved in the initiation of e.j.ps.

\section{Methods}

Experiments were carried out on mesenteric arteries and veins arising from the superior mesenteric artery or vein of guinea-pigs (male or female, 150-300 g). Animals were stunned and exsanguinated. Sections of small intestine with attached arcades of mesenteric arteries and veins were rapidly removed and placed in a dissecting dish containing physiological saline (composition mM: $\mathrm{NaCl} 120, \mathrm{KCl} 5, \mathrm{CaCl}_{2} 2.5$, $\mathrm{MgCl}_{2} 2.0, \mathrm{NaH}_{2} \mathrm{PO}_{4} 0.1, \mathrm{NaHCO}_{3} 25$ and glucose, 11 ; gassed with $95 \% \mathrm{O}_{2}$ and $5 \% \mathrm{CO}_{2}$ ).

\section{Electrical recordings}

Lengths $(1 \mathrm{~cm})$ of artery and adjacent vein were removed from the arcade and pinned side by side in a recording chamber (volume $0.2 \mathrm{ml}$ ). To obtain rapid changes in bath composition a rapid-action two-way valve (Polyplan. Glen Waverly, Vic.) was placed adjacent to the bath (see Hirst et al., 1982). In some experiments a larger volume chamber $(1 \mathrm{ml}$, see Hirst, 1977) was used to enable a stimulating electrode to be placed around the proximal ends of the artery and vein. Perivascular nerves were stimulated at supramaximal parameters $(20-60 \mathrm{~V}, 0.2-0.5 \mathrm{~ms}$ pulse width). Preparations were superfused at a rate of $6 \mathrm{ml} \mathrm{min}-1$ with gassed physiological saline warmed to $37^{\circ} \mathrm{C}$.
Intracellular recordings were made with microelectrodes pulled from fibre-containing glass tubes (Clark electromedical, GC150F-15) with BrownFlaming micropipette puller (model P.77, Sutter Instrument Co; San Francisco, California) and filled with $0.5 \mathrm{M} \mathrm{KCl}$. Electrodes with resistances in the range $80-160 \mathrm{M} \Omega$ were used. Recordings were digitized and stored on disc for later analysis. Data acquisition and analysis were performed using programmes written under the DAOS software package (version 7.0, Laboratory Software Associates).

\section{Mechanical recordings}

Lengths of artery and vein of $5 \mathrm{~mm}$ were used for tension records. Vessels were attached to two perspex 'feet' by means of $50 \mu \mathrm{m}$ tungsten wire (Goodfellow Metals, Cambridge) placed through the lumen. One foot was attached to a micromanipulator (Narashige model $\mathrm{mm}-3$ ) whilst the other was attached to a Kistler-Morse deflection sensor (DSC46.1003.01) for recording isometric changes in force. The voltage signal was amplified and filtered using a strain gauge conditioner (Analog devices model 2B31J) and the output was recorded on a pen recorder (Riken Denshi model SP-GC3). A resting tension of $500 \mathrm{mg}$ was maintained on arteries and $200 \mathrm{mg}$ on veins. Vessels were left to equilibrate for a period of $30 \mathrm{~min}$ before experiments were begun. Physiological saline, as used in the electrophysiological studies, was warmed to $35^{\circ} \mathrm{C}$ and flowed through a $5 \mathrm{ml}$ bath at $7 \mathrm{ml} \mathrm{min}^{-1}$.

Irreversible blockade of $\alpha$-adrenoceptors was achieved by perfusing preparations with $10 \mu \mathrm{M}$ benextramine (BNX) for $30 \mathrm{~min}$ followed by a $30 \mathrm{~min}$ wash period before recordings were made (Melchiorre et al., 1978; Hirst \& Lew, 1987).

Drugs used were: adenosine $5^{\prime}$ triphosphate (ATP), $\alpha-, \beta$-, methylene adenosine $5^{\prime}$ triphosphate (Me-ATP), benextramine hydrochloride, noradrenaline bitartrate ((-)-arterenol) (all obtained from Sigma chemicals), bretylium toxylate (Serva), desmethylimipramine (free base) (Ciba Geigy).

\section{Results}

\section{Responses to sympathetic nerve stimulation recorded from mesenteric arteries and veins}

The resting membrane potential of mesenteric arterial smooth muscle cells was $-66 \pm 0.6 \mathrm{mV}$ $(n=87)$. When arterial perivascular nerves were stimulated, e.j.ps were initiated. Single supramaximal stimuli evoked an e.j.p. with a peak amplitude of $3.9 \pm 0.4 \mathrm{mV}$, a rise time of $47.8 \pm 1.5 \mathrm{~ms}$ and a time constant of decay of $295 \pm 35.9 \mathrm{~ms}(n=6)$ (Figure 1a). E.j.ps were not associated with a visible contrac- 


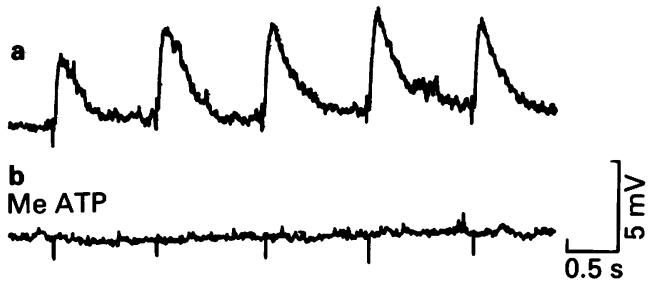

Figure 1 E.j.ps recorded from a mesenteric artery following sympathetic nerve stimulation. The record shown in (a) illustrates the response to successive supramaximal perivascular stimuli at a frequency of $1 \mathrm{~Hz}$. The lower record (b) shows that the e.j.ps are abolished during prolonged superfusion with a solution containing $10 \mu \mathrm{M}$ Me-ATP. Calibration bars apply to both records.

tion. When short trains of stimuli $(20 \mathrm{~Hz})$ were applied, e.j.ps summed together and triggered an action potential. This in turn initiated arterial constriction. The irreversible $\alpha_{1} / \alpha_{2}$-adrenoceptor antagonist benextramine $(10 \mu \mathrm{M})$ did not alter the

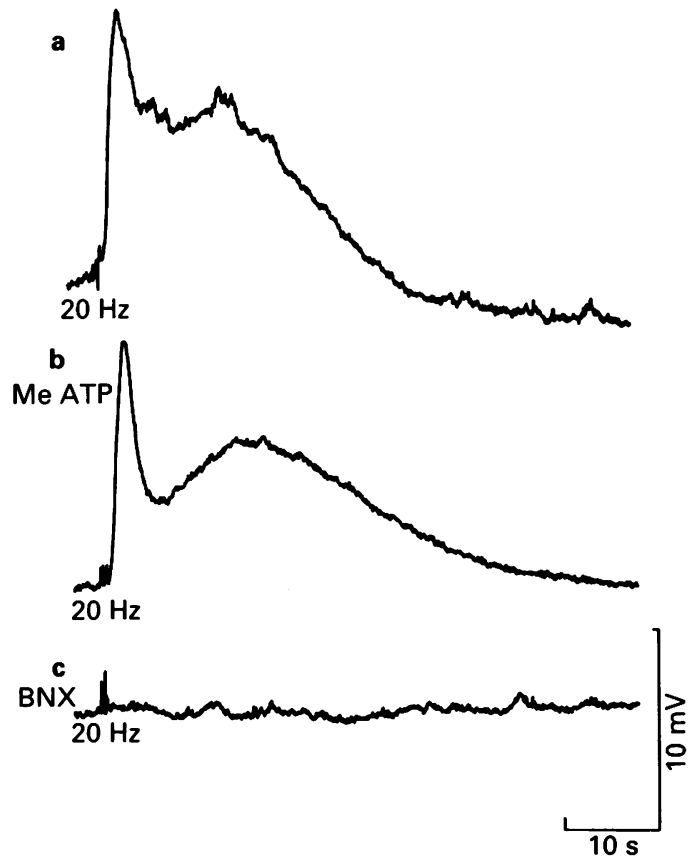

Figure 2 Depolarizations of mesenteric veins produced by stimulation of sympathetic nerves. In all three records, perivascular nerves were stimulated at a frequency of $20 \mathrm{~Hz}$ for $750 \mathrm{~ms}$ with supramaximal voltages. The record in (a) shows the effect of stimulation in control solution. The middle record (b) shows the depolarizations are little affected by prolonged incubation in $10 \mu \mathrm{M}$ Me-ATP. The lower record (c) shows that the response to nerve stimulation is abolished after incubation in $10 \mu \mathrm{M}$ benextramine (BNX).

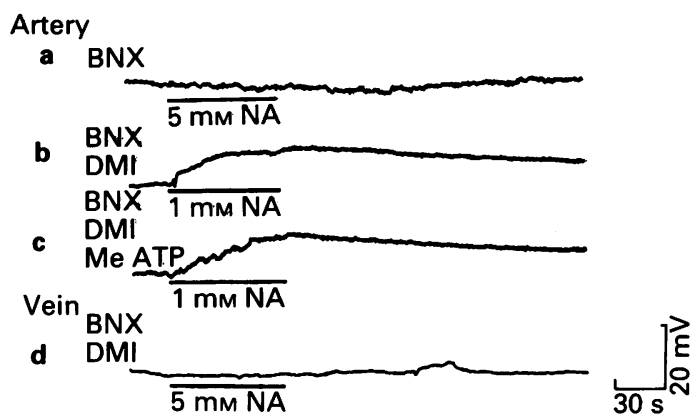

Figure 3 Noradrenaline (NA) responses in benextramine (BNX) treated mesenteric arteries and veins. Record (a) shows perfusion with NA after BNX treatment alone. The record in (b) shows that perfusion with the uptake inhibitor desmethylimipramine (DMI) reveals a depolarization in response to NA perfusion. Record (c) shows that incubation in Me-ATP has little effect on this depolarizing response to NA. Application of NA to veins in the presence of DMI (record d) produces little response. Perfusion times are shown by bars. Calibration bars apply to all records.

size of arterial e.j.ps (amplitude $=4.0 \pm 0.2 \mathrm{mV}$, $n=5$ ), whilst the adrenergic neurone blocker bretylium $(10 \mu \mathrm{M})$ abolished e.j.ps after approximately $30 \mathrm{~min}$.

Venous smooth muscle cells had a resting membrane potential of $-70 \pm 0.9 \mathrm{mV}(n=23)$. A single stimulus applied to the perivascular nerves of veins failed to initiate either a rapid e.j.p or any other detectable depolarization. Repetitive nerve stimulation $(>10 \mathrm{~Hz})$ caused a slow e.j.p. which usually showed two components, an initial peak followed by a slower depolarization (Figure 2a). At fixed frequencies this slow e.j.p. increased in amplitude with increasing stimulus voltages until a maximum was reached. Further amplitude increases could be achieved by then increasing stimulus frequency. Stimulation at $20 \mathrm{~Hz}$ for $750 \mathrm{~ms}$ at supramaximal voltages initiated a slow e.j.p. in which the initial component was larger with an amplitude of $8.9 \pm 1.9 \mathrm{mV}(n=6)$ (Figure 2a). The slow e.j.p. and contraction following venous nerve stimulation was abolished by $10 \mu \mathrm{M}$ benextramine $(n=4)$ (Figure $2 \mathrm{c}$ ).

Distribution of $\gamma$-adrenoceptors and $P_{2}$ receptors on mesenteric arteries and veins

Noradrenaline (1-10 $\mu \mathrm{M})$, applied by superfusion, caused contraction of both arteries and veins. In both tissues these responses were prevented by incubating the tissues in benextramine. In either tissue, increasing the concentrations of noradrenaline up to $5 \mathrm{~mm}$ failed to produce a detectable depolarization but occasionally produced a small hyperpolarization (Figure 3a). After the addition of the neuronal amine 


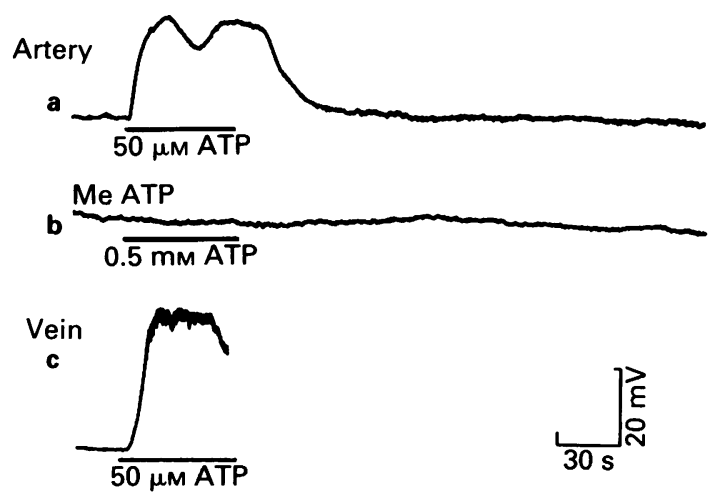

Figure 4 Effect of adenosine triphosphate (ATP) on the membrane potential of mesenteric arteries and veins. The upper record (a) shows a rapid depolarization in response to $50 \mu \mathrm{M}$ ATP. The middle record (b) shows that the response to $0.5 \mathrm{~mm}$ ATP is abolished by incubation in $10 \mu \mathrm{M}$ Me-ATP. The lower record (c) shows a rapid depolarization to $50 \mu \mathrm{M}$ ATP in a vein. Bars indicate perfusion time. Calibration bars apply to all records.

uptake blocker desmethylimipramine (DMI) $(1 \mu \mathrm{M})$, noradrenaline $(>0.2 \mathrm{mM})$ caused arterial depolarization and contraction (Figure $3 b$ ). The amplitude of depolarization varied between preparations and electrodes were often dislodged due to contraction. The mean depolarization produced by noradrenaline $(0.5 \mathrm{mM})$ was $12.6 \pm 1.4 \mathrm{mV}(n=12)$.

Application of very high concentrations of noradrenaline to veins after benextramine treatment did not lead to a membrane potential change $(n=4)$. However, unlike arteries the addition of desmethylimipramine did not reveal an $\alpha$-antagonist-resistant response (Figure 3d).

ATP $(>0.1 \mathrm{~mm})$ caused substantial membrane depolarization in both arteries and veins (Figure $4 \mathrm{a}, \mathrm{c})$ with $0.5 \mathrm{mM}$ ATP causing $18 \pm 4 \mathrm{mV}(n=5)$ depolarization in arteries and $23 \pm 4 \mathrm{mV}(n=3)$ for veins. These depolarizations often caused action potentials and were associated with rapid contractions which usually dislodged the electrodes.

\section{Mechanical responses}

Contraction of arteries to exogenous noradrenaline had a threshold of $5 \mu \mathrm{M}$ (Figure 5a). After benextramine treatment in the presence of desmethylimipramine, arteries proved unresponsive to even high concentrations (1 mM) of noradrenaline if the concentration was allowed to rise slowly. Rapid contractions were produced if the bath concentration of noradrenaline was rapidly increased to its final concentration $(0.5-2 \mathrm{~mm})$ by microinjection into the bath (Figure 5a) (cf. Byrne \& Large, 1986). Contractions produced by $1 \mathrm{~mm}$ noradrenaline were $1099 \pm 252 \mathrm{mg},(n=6)$. ATP $(1 \mathrm{mM})$ also produced a rapid contraction although never as large as that to noradrenaline (678 $\pm 135 \mathrm{mg}, n=5$ ) (Figure 5a).

Veins contracted with much less force than arteries. The maximal contraction caused by increasing the potassium concentration to $70 \mathrm{~mm}$ was $254 \pm 26 \mathrm{mg}, \quad n=8$ (Figure $5 \mathrm{~b}$ ). Noradrenaline $(0.1 \mathrm{mM})$ caused a contraction of $127 \pm 18 \mathrm{mg}(n=7)$ (Figure 5b). This contraction was abolished by incubation in $10 \mu \mathrm{M}$ benextramine. After incubation in benextramine and in the presence of DMI, noradrenaline in concentrations of up to $2 \mathrm{mM}$ added by microinjection, failed to produce a detectable contraction (Figure 5b). ATP (1 mM) caused a contraction in all venous preparations, the mean peak tension ( $247 \pm 41 \mathrm{mg}, n=8)$ was similar to that produced during a maximal potassium contraction (Figure 5b).

\section{Effects of Me-ATP}

Perfusing the preparation with $10 \mu \mathrm{M}$ Me-ATP caused a rapid depolarization of arteries and veins. After $30 \mathrm{~min}$ the vessels had repolarized to control values. Measurements of tension showed that Me-ATP 10 and $100 \mu \mathrm{M}$ produced a rapid transient contraction which soon returned to a stable resting tension (Figure 5a). In the presence of Me-ATP, sympathetic nerve stimulation now failed to produce an e.j.p. in the artery. However, the slow e.j.p. detected in veins after sympathetic nerve stimulation was little affected (mean peak amplitude $7.6 \pm 2.9 \mathrm{mV}, n=5$ ) (Figure 2b).

The benextramine-resistant depolarization of arteries, ( $\gamma$-adrenoceptor response), was unaffected by the presence of $10 \mu \mathrm{M} \mathrm{Me}-\mathrm{ATP}$, whilst the response to ATP $(0.5 \mathrm{mM})$ was abolished $(n=4)$ (Figure $4 \mathrm{~b})$.

Tension measurements in arteries showed that the benextramine-resistant peak contraction to noradrenaline was unchanged $(1393 \pm 99 \mathrm{mg}, n=3)$ whilst the response to ATP was greatly attenuated $31.75 \pm 31 \mathrm{mg}(n=3)$ (Figure 5).

\section{Discussion}

This study compared the membrane potential changes that occur in mesenteric arteries and veins during sympathetic transmission. The distributions of $\mathbf{P}_{2}$ purinoceptor and $\gamma$-adrenoceptor types on these vessels were also determined. As has been shown previously, rapid e.j.ps were initiated in mesenteric arteries (Kuriyama \& Suzuki, 1981; Suzuki, 1981; Ishikawa, 1985; Kreulen, 1986). Rapid e.j.ps persisted after treatment with the irreversible $\alpha$ adrenoceptor antagonist, benextramine, but were abolished by bretylium, which prevents the release of 


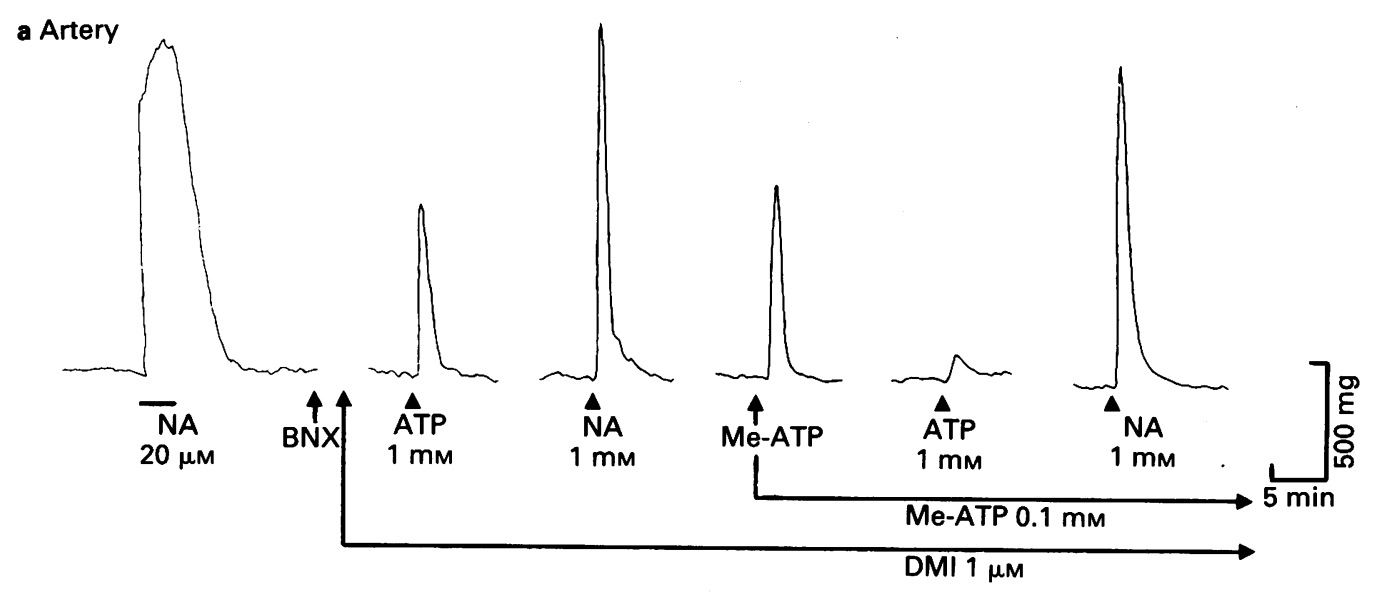

b Vein

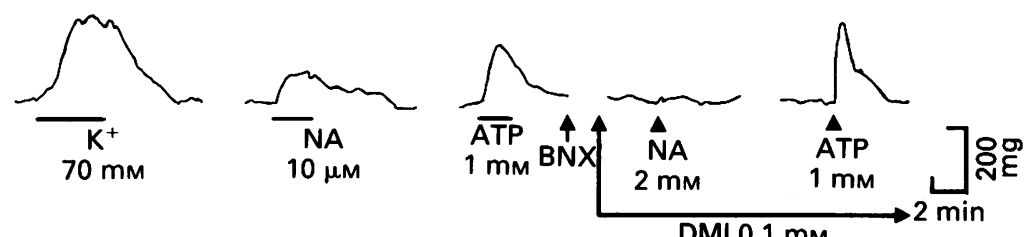

Figure 5 The effect of noradrenaline (NA) and ATP on tension in mesenteric arteries and veins. The upper trace (a) is of an artery showing that the contraction produced by NA after benextramine (BNX) treatment requires a relatively high NA concentration and is little affected by incubation in $0.1 \mathrm{mM}$ Me-ATP, whilst the response to ATP is almost abolished. The preparation was continuously perfused with $1 \mu \mathrm{M}$ desmethylimipramine (DMI) following BNX treatment. Continuous perfusion with Me-ATP caused an initial contraction which relaxed to resting levels. The lower trace (b) is of a vein showing the presence of $\alpha$-adrenoceptors and $P_{2}$ purinoceptors but an absence of response to NA after BNX treatment. The preparation was continuously perfused with DMI following BNX treatment. Bars indicate perfusion times. Arrow heads $(\Delta)$ indicate microinjection into the organ bath. The arrows below the trace indicate continuous perfusion of Me-ATP or DMI.

noradrenaline from sympathetic nerves (Bowman \& Rand, 1980). In contrast, rapid e.j.ps were not recorded from mesenteric veins following sympathetic nerve stimulation, rather repetitive nerve stimulation initiated a slow long lasting membrane depolarization. This slow e.j.p. was associated with constriction and was prevented by benextramine. Similar observations have been made from a number of venous preparations (Suzuki, 1981; Kreulen, 1986; van Helden, 1988).

Both arteries and veins contracted when low concentrations of noradrenaline were applied. These responses were abolished by benextramine treatment. Neither arterial nor venous preparations responded to high concentrations of noradrenaline after benextramine treatment. However when the tissues were further incubated with DMI, an inhibitor of neuronal uptake, the arteries alone responded to noradrenaline. At these concentrations of noradrenaline, Uptake ${ }_{1}$ should be saturated (Lightman $\&$ Iversen, 1969) and inhibition of uptake would be expected to have no effect on the concentration of noradrenaline reaching the receptor. The simplest explanation of this is that the $\gamma$-adrenoceptors activated by exogenous noradrenaline lie next to an uptake site which is not saturated. This could occur if $\gamma$-adrenoceptors are localised near the sympathetic neuromuscular junction and diffusional access to noradrenaline is limited by a narrow junctional gap. This suggestion is in accord with the observation that $\gamma$-adrenoceptors are restricted to regions of the artery near sympathetic varicosities (Hirst \& Neild, 1981; Luff, 1987).

The lack of response of veins to noradrenaline after $\alpha$-adrenoceptor blockade even in the presence of DMI suggests that they are devoid of $\gamma$ adrenoceptors. Tension studies on a variety of rabbit blood vessels found that non- $\alpha$-adrenoceptors were restricted to systemic arteries but not veins (Laher $e$ t $a l ., 1986)$. The distribution of $\gamma$-adrenoceptors, present in arteries but not veins, is therefore correlated with the presence of $\alpha$-antagonist resistant 
neuronal responses. A similar finding has been made in rat middle cerebral arteries where $\gamma$-adrenoceptors were found in proximal innervated arterioles but not in the distal arterioles which never receive an innervation (Edwards et al., 1989).

Both arteries and veins responded to ATP. Therefore the distribution of $P_{2}$ purinoceptors shows no correlation with the ability of sympathetic nerves to initiate an e.j.p. If sympathetic nerves did indeed release appreciable amounts of ATP, then non- $\alpha$ adrenoceptor neuronal responses should be detected in veins unless the junctional cleft width is large or innervation is sparse. Where measured, the junctional cleft width in veins has been shown to be similar to that of arteries and often narrow (Rowan \& Bevan, 1983). Mesenteric veins of the guinea-pig have been shown to be well innervated and although they appear less dense in wholemounts than arteries (Furness, 1971), the medial layer is much thinner and nerve density may be equivalent. Given a similar neuromuscular junction to the artery, either the sympathetic nerves which innervate veins must fail to release ATP, or $P_{2}$ purinoceptors may be located away from points of transmitter release. $P_{2}$ purinoceptors are relatively low affinity receptors and may only be activated in a junctional position where the local concentration of transmitter is high.

The slow e.j.p. in veins was not seen until stimulation frequency was increased. Similarly, at the low stimulus parameters required to activate arterial e.j.ps, no responses attributable to $\alpha$-adrenoceptors were found. However, relatively high frequency stimulation has been reported to activate $\alpha$ adrenoceptors in these arteries (Hottenstein \& Kreulen, 1987). Such a dependence on high frequencies could indicate an extrajunctional position for $\alpha$-adrenoceptors.

In rat basilar arteries, Me-ATP has been shown to be an antagonist to both $\gamma$-adrenoceptors and $\mathbf{P}_{2}$ purinoceptors (Byrne \& Large, 1986). This clearly is not the case for mesenteric vessels. Me-ATP abolished the responses produced by ATP in both

\section{References}

BENHAM, C.D. \& TSIEN, R.W. (1987). Noradrenaline increases L-type calcium current in smooth-muscle cells of rabbit ear artery independently of $\alpha$ - and $\beta$ adrenoceptors. J. Physiol., 390, 85P.

BOWMAN, W.C. \& RAND, M.J. (1980). Textbook of Pharmacology. London: Blackwell.

BURNSTOCK, G. \& WARLAND, J.J.I. (1987). A pharmacological study of the rabbit saphenous artery in vitro: a vessel with a large purinergic contractile response to sympathetic nerve stimulation. Br. J. Pharmacol., 90, 111-120. arteries and veins but did not abolish the responses produced by $\gamma$-adrenoceptor activation in mesenteric arteries. The different sensitivity of $\gamma$-adrenoceptors in the rat basilar artery (Byrne \& Large, 1986) and those of the guinea-pig mesenteric artery to Me-ATP, suggests that more than one class of $\gamma$ adrenoceptors may exist. The proposal of more than one $\gamma$-adrenoceptor has been previously suggested for the rat basilar artery where a biphasic depolarization to exogenous noradrenaline exists, neither component being due to $\alpha$-adrenoceptor activation (Byrne et al., 1986). The first component of this depolarization was easily desensitized and was only seen if noradrenaline was micro-injected into the bath. That is, if the bath concentration rose quickly (Byrne et al., 1986). No biphasic $\gamma$-adrenoceptor response could be detected in this study even though the bath concentration would have risen quickly (see Methods).

Since Me-ATP abolished the rapid e.j.ps recorded from arteries, it seems unlikely that the activation of $\gamma$-adrenoceptors can underlie the initiation of an e.j.p. Thus arteries have a population of adrenoceptors that are located near sympathetic nerve terminals and are only activated by high concentrations of noradrenaline. It has already been pointed out that arteries generate muscle action potentials during neuromuscular transmission. Since the ability of arterioles to support inward calcium movement is related to the density of sympathetic nerves (Hill et al., 1986), it could be that $\gamma$-adrenoceptors are closely linked to voltage-dependent calcium channels. This suggestion has been previously made by Benham \& Tsien (1987), who found that calcium entry into single isolated arterial cells was increased in the presence of noradrenaline, the receptor involved was neither an $\alpha$-nor a $\beta$-adrenoceptor.

\section{Acknowledgement}

This project was supported by a grant from the $\mathrm{NH}$ and MRC. We thank them for this financial support.
BYRNE, N.G. \& LARGE, W.A. (1986). The effect of $\alpha, \beta$ methylene ATP on the depolarization evoked by noradrenaline ( $\gamma$-adrenoceptor response) and ATP in the immature rat basilar artery. Br. J. Pharmacol., 88, 6-8.

BYRNE, N.G., HIRST, G.D.S. \& LARGE, W.A. (1985). Electrophysiological analysis of adrenoceptors in the rat basilar artery during development. Br. J. Pharmacol., 86, 217-227.

CHEUNG, D.W. (1981). An electrophysiological study of $\alpha$ adrenoceptor mediated excitation-contraction coupling in the smooth muscle cells of the rat saphenous vein. $B r$. 
J. Pharmacol., 84, 265-271.

CHEUNG, D.W. (1982). Two components in the cellular response of the rat tail artery to nerve stimulation. $J$. Physiol., 328, 461-468.

EDWARDS, F.R., HARDS, D., HIRST, G.D.S. \& SILVERBERG, G.D. (1989). The localization of gamma-adrenoceptors and $P_{2}$ purine receptors on the rat cerebral arterioles. Br. J. Pharmacol. (in press).

FUJIWARA, S., ITOH, T. \& SUZUKI, H. (1982). Membrane properties and excitatory neuromuscular transmission in the smooth muscle of the dog cerebral arteries. $\mathrm{Br}$. J. Pharmacol., 77, 197-208.

FURNESS, J.B. (1971). The adrenergic innervation of the vessels supplying and draining the gastrointestinal tract. Z. Zellforsch., 113, 67-82.

HILL, C.E., HIRST, G.D.S., SILVERBERG, G.D. \& VAN HELDEN, D.F. (1986). Sympathetic innervation and excitability of arterioles originating from the rat middle cerebral artery. J. Physiol., 371, 305-316.

HIRST, G.D.S. (1977). Neuromuscular transmission in arterioles of guinea-pig submucosa. J. Physiol., 273, 263-275.

HIRST, G.D.S. \& EDWARDS, F.R. (1988). Sympathetic neuroeffector transmission in arteries and arterioles. Physiol. Rev., (in press).

HIRST, G.D.S. \& LEW, M.J. (1987). Lack of involvement of $\alpha$-adrenoceptors in sympathetic neural vasoconstriction in the hindquarters of the rabbit. Br. J. Pharmacol., 90, $51-60$.

HIRST, G.D.S. \& NEILD, T.O. (1980). Evidence for two populations of excitatory receptors for noradrenaline on arteriolar smooth muscle. Nature, 283, 767-768.

HIRST, G.D.S. \& NEILD, T.O. (1981). Localization of specialized noradrenaline receptors at neuromuscular junctions on arterioles of the guinea-pig. J. Physiol., 313, $343-350$.

HIRST, G.D.S., NEILD, T.O. \& SILVERBERG, G.D. (1982). Noradrenaline receptors on the rat basilar artery. $J$. Physiol., 328, 351-360.

HOLMAN, M.E. \& SUPRENANT, A.M. (1979). Some properties of the excitatory junction potentials recorded from saphenous arteries of rabbits. J. Physiol., 287, 337-351.

HOLMAN, M.E. \& SURPRENANT, A.M. (1980). An electrophysiological analysis of the effects of noradrenaline and $\alpha$-receptor antagonists on neuromuscular transmission in mammalian muscular arteries. Br. J. Pharmacol., 71, 651-661.

HOTTENSTEIN, O.D. \& KREULEN, D.L. (1987). Comparison of the frequency dependence of venous and arterial responses to sympathetic nerve stimulation in guineapigs. J. Physiol., 384, 154-157.

ISHIKAWA, S. (1985). Actions of ATP and $\alpha, \beta$-methylene ATP on neuromuscular transmission and smooth muscle membrane of the rabbit and guinea-pig mesenteric arteries. Br. J. Pharmacol., 86, 777-787.

KREULEN, D.L. (1986). Activation of mesenteric arteries and veins by preganglionic and postganglionic nerves. Am. J. Physiol., 251, H1267-H1275.
KUGELGEN, I.V. \& STARKE, K. (1985). Noradrenaline and adenosine triphosphate as co-transmitters of neurogenic vasoconstriction in rabbit mesenteric arteries. $J$. Physiol., 367, 435-455.

KURIYAMA, H. \& SUYAMA, A. (1983). Multiple actions of cocaine on neuromuscular transmission and smooth muscle cells of the guinea-pig mesenteric artery. $J$. Physiol., 337, 631-654.

KURIYAMA, H. \& SUZUKI, H. (1981). Adrenergic transmissions in the guinea-pig mesenteric artery and their cholinergic modulations. J. Physiol., 317, 383-396.

LAHER, I., KHAYAL, M.A. \& BEVAN, J.A. (1986). Norepinephrine sensitive, phenoxybenzamine-resistant receptor sites associated with contraction in rabbit arterial but not venous smooth muscle; possible role in adrenergic neurotransmission. J. Pharmacol. Exp. Ther., 237, 364-368.

LIGHTMAN, S. \& IVERSEN, L.L. (1969). The role of uptake in the extraneuronal metabolism of catecholamines in the isolated rat heart. Br. J. Pharmacol., 37, 638-649.

LUFF, S.E., MACLACHLAN, E.M. \& HIRST, G.D.S. (1987). An ultrastructural analysis of the sympathetic neuromuscular junctions on arterioles of the submucosa of the guinea pig ileum. J. Comp. Neurol., 257, 578-594.

MELCHIORRE, C., YONG, M.S., BENFEY, B.G. \& BELLEAU, B. (1978). Molecular properties of adrenergic alphareceptor. 2. Optimum covalent inhibition by two different prototypes of polyamine disulfides. J. Med. Chem., 21, 1126-1132.

MURAMATSU, I. (1986). Evidence for sympathetic, purinergic transmission in the mesenteric artery of the dog. Br. J. Pharmacol., 87, 478-480.

ROWAN, R.A. \& BEVAN, J.A. (1983). Distribution of adrenergic synaptic cleft width in vascular and non-vascular smooth muscle. In Vascular Neuroeffector Mechanisms. 4th International Symposium. ed. Bevan, J.A. pp. 75-93. New York: Raven Press.

SNEDDON, P. \& BURNSTOCK, G. (1984). ATP as a cotransmitter in rat tail artery. Eur. J. Pharmacol., 106, 149-152.

SURPRENANT, A. (1980). A comparative study of neuromuscular transmission in several mammalian muscular arteries. Pflugers Archiv., 386, 85-91.

SUZUKI, H. (1984). Adrenergic transmission in the dog mesenteric vein and its modulation by $\alpha$-adrenoceptor antagonists. Br. J. Pharmacol., 81, 479-489.

SUZUKI, H. (1981). Effects of endogenous and exogenous noradrenaline on the smooth muscle of guinea-pig mesenteric vein. J. Physiol., 321, 495-512.

SUZUKI, H. \& KOU, K. (1983). Electrical components contributing to the nerve-mediated contractions in the smooth muscles of the rabbit ear artery. Jpn. J. Physiol., 33, 743-756.

vAN HELDEN, D.F. (1988). Electrophysiology of neuromuscular transmission in guinea-pig mesenteric veins. $J$. Physiol., 401, 469-488. 